\title{
EVALUACIÓN DEL EFECTO DE LA TEMPERATURA SOBRE EL CRECIMIENTO DE CULTIVOS INICIADORES DURANTE LA MADURACIÓN DEL QUESO TIPO EDAM
}

\author{
EVALUATION OF THE EFFECT OF TEMPERATURE ON THE GROWTH OF \\ MOULD STARTERS DURING THE MATURATION OF EDAM CHEESE TYPE
}

${ }^{1}$ Edhy Teodoro León Hancco ; ${ }^{2}$ Thomas Ancco Vizcarra; ${ }^{3}$ Juan Marcos Aro Aro; ${ }^{4}$ Marienela Calsin Cutimbo

\section{RESUMEN}

Este estudio se realizó en Puno, ubicado a 3824 m.s.n.m. Se evaluó el pH y acidez, además de la validación de los modelos matemáticos de crecimiento de cultivos iniciadores del género Lactococcus RSF-742 (CHR HANSEN) a temperaturas 30,38 y 45 ${ }^{\circ} \mathrm{C}$ en función del tiempo, en la maduración del queso Edam. Los datos de crecimiento fueron ajustados a modelos: Baranyi \& Roberts, Gompertz y Logístico para determinar los parámetros $\left(A, b, C y D, \lambda, \operatorname{Tg} y \mu_{\text {ma }}\right)$. La temperatura $30^{\circ} \mathrm{C}$ fue el factor que presentó mayor efecto sobre los parámetros: $A=5,95 ; b=0,22 ; C=8,48$ y $D=52,82$ y los parámetros cinéticos: $\lambda=48,35, T g=$ 0,63 y $\mu=0,69$ horas. Finalmente la evolución de $\mathrm{pH}$ y acidez fueron evaluadas obteniendo los resultados a temperatura $30 \mathrm{y}$ $38^{\circ} \mathrm{C}$ con valores óptimos de pH: 5,92 y 6,05 y acidez: 0,091 y 0,054 respectivamente en maduración del queso Edam. De la validación a 30, 38 y $45^{\circ} \mathrm{C}$ se determinó que el modelo Gompertz predice mejor (los indices de factor sesgo $(B f)=$ factor exactitud $(A f)=1,00)$, seguido de Baranyi \& Roberts $(B f=A f=1,01)$ y Logístico $(B f=0,99$ y $A f=1,00)$. Por lo tanto los modelos validados corresponden a Baranyi \& Roberts y Gompertz.

Palabras Clave: queso Edam, modelo, factor sesgo, factor exactitud.

\section{ABSTRACT}

The study was developed in Puno, located at 3824 above sea level; the pH and acidity plus the validation of mathematical models of mould starters growing crops of the genus Lactococcus RSF-742 (CHR HANSEN) at temperatures of 30,38 and $45^{\circ} \mathrm{C}$ versus Edam cheese ripening time were evaluated. Growth data were fitted to models: Baranyi \& Roberts, Gompertz and Logistic to determine the parameters $\left(A, b, C y D, \lambda, T g y \mu_{m}\right)$. The $30^{\circ} \mathrm{C}$ temperature was the factor that had a greater effect on parameters: $a=5.95, b=0.22, C=8.48$ and $D=52.82$ and kinetic parameters. $\lambda=48.35, g=0.63$ and $\mu_{\operatorname{man}}=0.69$ hours. Finally the evolution of pH and acidity were evaluated obtaining results at temperature $3038^{\circ} \mathrm{C}$ with optimum pH values: 5.92 and 6.05 and acidity: 0.091 and 0.054 , validation 30,38 and $45^{\circ}$ C.It was determined that the Gompertz model predicts better (rates bias factor $(B f)=$ accuracy factor $(A f)=1.00)$, followed by Baranyi \& Roberts $(B f=A f=1.01)$ and Logistic $(B f=0.99$ and $A f=1.00)$. Therefore the validated models correspond to Gompertz and Baranyi \& Roberts.

Key Words: Edam cheese, model, bias factor, accuracy factor:

\section{INTRODUCCIÓN}

El queso Edam se procesa con pasta lavada y alto grado de mineralización, presenta buena flexibilidad al tajado, es de gusto suave, pocos ojos ovalados, lisos regularmente distribuidos y su sabor es más suave que el Gouda (Ramirez, 2005). Su maduración requiere 3 a 4 semanas a $12 \sim 14^{\circ} \mathrm{C}$, el periodo de maduración depende del contenido de humedad al que se desee ajustar (Scott, et al., 2002 ), humedad relativa, $80 \sim 85 \%$, temperatura; $8 \sim 12^{\circ} \mathrm{C}$ y ventilación media (Ramirez, 2005). Dentro de los factores que influencian se tiene a la temperatura que incide en la velocidad de maduración, pues afecta la actividad microbiana y enzimática (Ramirez, 2005). El valor de $\mathrm{pH}$ alcanzado en un queso durante el proceso de elaboración y de maduración es $\mathrm{pH} 5,8 \sim 6,5$. Las primeras fases de fabricación del queso son las que determinan la velocidad de producción de la acidez, alcanzándose el $\mathrm{pH}$ para cada variedad de queso (Hill, 2007); el pH regula la actividad bacteriana y enzimática, reduciendo a $\mathrm{pH} 5 \sim 5,2$, en la cuajada fresca, rápidamente en las primeras horas, se mantiene estable por un tiempo y luego comienza a ascender

Ingeniero Agroindustrial, Facultad de Ingenicria Agroindustrial de la Universidad Nacional del Altiplano. Petao-Perú.

Magister Scientiae en Postcosecha y Marketing. Ingeniero Agroindustrial. Facultad de Ingenieria Agroindustrial de la Universidad Nacional José Maria Aryuedas. Apurimac-Perü. "Doctor of Philosophy, Magister Seientiae en Tecnologia de Alinentos. Facultad de Ingenieria Agroindustrial de la Universidad Nacional del Altiplano. Puno-Perù.

Ingeniero Agroindustrial. Facultad de Ingenieria Agroindustrial de La Universidad Naciunal del Altiplano. Puno-Penù. 
paulatinamente en ciertos quesos, donde puede llegar a valores de 5,4 5,6 o fuertemente en otros (madurados por mohos y levaduras) y que llega a neutralidad (Condori, 2010). Durante la maduración los quesos pierden peso por evaporación y desarrollan aromas y sabores característicos de cada tipo (Madrid, 1994). Los componentes del queso que se modifican durante el curado son: la grasa, la proteína y la lactosa. La grasa se elimina con la fracción del suero y la cantidad que se retiene en la cuajada se fermenta formando ácido láctico, durante la maduración la fracción de proteínas se sujeta a la acción de microorganismos y enzimas y se hidroliza parcialmente. Los cultivos iniciadores constituyen una parte importante de una industria plenamente desarrollada y son en muchas ocasiones, condición indispensable para la fabricación de gran variedad de productos lácteos fermentados, los cultivos iniciadores se definen como cultivos de una o varias cepas, pertenecientes a una o várias especies de bacterias deseables, que se utilizan para inocular leche pasteurizada o cruda, fundamentalmente con el objeto de iniciar la fermentación (Sandine, 1979). El grupo más importante está integrado casi exclusivamente por bacterias ácido lácticas (BAL) pertenecientes a los géneros Lactococcus, Lactobacillus y Leuconostoc. Además de $\mathrm{BAL}$, en algunas ocasiones se añaden a los fermentos para la fabricación de queso, los llamados cultivos secundarios o adjuntos, constituidos también por bacterias o por mohos y levaduras, que actúan durante la maduración produciendo compuestos que intervienen en el aspecto, textura, sabor y aroma de los mismos, por lo que la principal función de los cultivos iniciadores es la producción de ácido láctico por fermentación de la lactosa presente en la leche, creando unas condiciones favorables: i) Favorecer la formación de la cuajada por enzimas coagulantes. ii) Prevenir o inhibir el crecimiento de la flora patógena y alterante mediante la reducción del $\mathrm{pH}$. iii) Contribuir a la formación de la textura y compuestos del sabor y aroma característicos (Ayad, 2000). Los cultivos iniciadores están compuestos por cepas de Lactococcus lactis subsp. lactis, L. lactis subsp. cremoris, L. lactis subsp. lactis biovar diacetylactis y Leuconostoc. Por otro lado, los cultivos termófilos contienen Streptococcus thermophilus y diferentes especies de lactobacilos como Lactobacillus delbrweckii subsp. lactis, $L$ b. belveticus y $L$ b. fermentum, (Pavlidou et al., 2011). Los fermentos lácticos actúan en el queso desde que comienza la fabricación hasta el final de la maduración. La primera acción es la acidificación rápida del medio que permite controlar y frenar el desarrollo de la flora heterogénea natural de la leche y desciende el $\mathrm{pH}$ favoreciendo la actividad coagulante del cuajo (Oria, 1991) y (Holzapfel, 2002). Los microorganismos más importantes para la industria láctea son 10 géneros: Corynebacterium, Enterococcus, Lactococcus, Lactobacillus, Leuconostoc, Oenococcus, Pediococcus, Streptococcus, Tetragenococcus y Vagococcus (Janes \& Martin, 2009).

\section{Validación de los modelos matemáticos}

La validación de un modelo matemático es una de las etapas más cruciales y al mismo tiempo más dificil dentro del ciclo completo de la modelización predictiva. (Van Boekel, 1996). La validación debe realizarse en medios de laboratorio pero especialmente en productos alimenticios.
Cuando el sistema, al que se aplica un modelo predictivo, se hace más complejo, aumenta el error de las predicciones (Pin et al., 1999), la validación de modelos predictivos se puede llevar a cabo con datos obtenidos en las mismas condiciones que los utilizados para elaborar el modelo, denominada validación interna, con el fin de determinar si el modelo puede describir suficientemente los datos experimentales (Castillejo, 2002).

La validación matemática cuantifica la seguridad matemática de un modelo testado con datos experimentales nuevos (Van Boekel, 1996). La proximidad en el valor del error cuadrático medio (MSE, Mean Square Error) y los valores de $\mathrm{R}^{2}$, de las ecuaciones ajustadas a la serie de datos, se han tomado como indicadores de la frabilidad de los modelos cuando se aplicaban a alimentos. Otra medida de la exactitud de las ecuaciones predictivas fue introducida por (McClure et al., 1993) quienes compararon sus modelos basándose en el sumatorio de los cuadrados de las diferencias del logaritmo natural de los valores observados y predictivos para el tiempo de generación (Lima, 2010).

$$
\sum\left(\operatorname{Ln}\left(\mathrm{GT}_{\text {observado }}\right)-\operatorname{Ln}\left(G T_{\text {predictivo }}\right)\right)^{2}
$$

El valor más pequeño indica el modelo que mejor predice la respuesta observada. Ross (1996) ha desarrollado dos índices para evaluar la bondad de ajuste de los modelos predictivos. Estos índices, denominados factor sesgo $(B)$ y exactitud $\left(A_{i}\right)$, proporcionan un fácil resumen de la fiabilidad de los modelos y además sirven como primer paso en el avance del proceso de validación de modelos predictivos. El objetivo planteado para estos índices fue permitir la estimación de la bondad de ajuste de modelos predictivos, cuando se comparan con observaciones no utilizadas para generar el modelo, particularmente llevadas a cabo en alimentos, y de esta forma evaluar su utilidad para ayudar en la toma de decisiones en seguridad y calidad alimentaria. Un objetivo adicional propuesto fue el de proporcionar una medida simple y cuantitativa de la fiabilidad de los modelos. Desde que Ross (1996) estableciera los índices, para evaluar la bondad de ajuste de los modelos predictivos, han sido ampliamente utilizados en estudios de validación (Neumeyer y col., 1997; Giffel y Zwietering, 1999; Ross y col., 2003; Barco, 2001; Castillejo, 2002).

El estadístico Raíz del error cuadrático medio (RMSE, Root Mean Square Error), raiz del sumatorio de los residuos al cuadrado dividido por el número de grados de libertad $(n)$, es una medida de variabilidad residual, la cual no es explicada por los cambios deliberados en factores como la temperatura, $\mathrm{pH}$ y $a_{*}$. El valor más bajo de RMSE significa la mejor idoneidad del modelo predictivo para describir los datos (Sutherland, et al., 1994).

$$
R M S E=\sqrt{\frac{\sum\left(\mu_{E s t}-\mu_{o b s}\right)^{2}}{n-p}}
$$

El coeficiente de regresión múltiple es utilizado con frecuencia como una medida de ajuste total, este coeficiente mide la fracción de la variación, alrededor de la media, que es explicada por el modelo. El valor más cercano 
a 1 significa una mejor predicción obtenida por ese modelo (Sutherland et al., 1994).

El índice sesgo y exactitud proporcionan una indicación objetiva de la idoneidad o buen funcionamiento de un modelo (Ross, 1996). En contraposición a los métodos estadísticos tradicionales, estos índices no están basados en la desviación existente entre la respuesta observada y la media. Este hecho causa un problema a la hora de evaluar la fiabilidad de los modelos con nuevos datos, puesto que la principal respuesta es desconocida. Está bien documentado que estos factores son herramientas útiles para medir la fiabilidad de los modelos predictivos (Neumeyer et al., 1997).

\section{La Bias o factor sesgo $(B)$}

Este factor responde a la interrogante ¿dónde se colocan los valores observados por encima o por debajo de la línea de equivalencia, y también en qué medida se acerca a la concordancia perfecta? Además proporciona una medida de la desviación estructural de un modelo. Está descrito en la ecuación siguiente:

$$
B_{f}=10\left[\frac{\sum \log \left(\frac{\mu_{E s t}}{\mu_{o b s}}\right)}{n}\right]
$$

Donde: : número de observaciones usadas en el calculo $\mu_{\text {fist }} \quad$ :Velocidad de crecimiento estimado/horas $\mu_{\text {Lis }} \quad$ : velocidad de crecimiento observado/ horas

Un factor sesgo $>1$ indica un modelo seguro para el caso de la tasa específica de crecimiento cuyo valor observado es inferior al estimado por el modelo predictivo, en este sentido el modelo proporciona un margen de seguridad (Ross, 1996). En cambio un valor sesgo $<1$ para la fase de adaptación proporcionado por un modelo significa que la predicción estimada es segura, puesto que la fase de adaptación observada es mayor que la predictiva. El factor sesgo no proporciona información del promedio de la exactitud de las estimaciones, puesto que la sobre o subestimación tiende a cancelarla. Por lo tanto se debe calcular el factor de exactitud (Cabeza, 2013).

\section{Accuracy o factor de exactitud (Af):}

Este factor es un promedio de distancia entre cada punto y la línea de equivalencia, como una medida de la proximidad del promedio entre las predicciones y las observaciones. Toma la siguiente forma en valor absoluto (Cabeza, 2013):

$$
A_{f}=10\left[\frac{\sum \log \left(\frac{\mu_{E s t}}{\mu_{v b s}}\right) \mid}{n}\right]
$$

Donde:

$$
\begin{array}{ll}
n & : \text { Número de observaciones } \\
p & : \text { Número de parámetros a ser estimados } \\
\mu_{\text {Ed }} & : \text { Velocidad de crecimiento estimada/hora } \\
\mu_{\text {Ots }} & : \text { Velocidad de crecimiento observado/hora. }
\end{array}
$$

El valor mayor alcanzado por el $A_{f}$ significa menos exactitud en la estimación. Un valor de $A$ de 1,7 indica que las predicciones, en promedio, difieren un $70 \%$ de las observaciones. Si no hay desviación estructural (sesgo $=1$, ambas desviaciones positiva y negativa en promedio, el modelo es exacto), la falta de exactitud pueden ser mostrada por el $A_{f}$ (Ross, 1996). Cuando ambos índices toman el valor de la unidad significa que existe concordancia perfecta entre los valores de los parámetros de crecimiento predictivos y los observados. Una buena concordancia entre los valores predictivos de tasa de crecimiento de un microorganismo y los observados en un alimento indica que el alimento por sí mismo juega un mínimo papel en la determinación de la respuesta microbiana, en otros casos la estructura del alimento juega un papel determinante, quizás porque limite la difusión de substratos u oxígeno al microorganismo o por producción de compuestos ácidos (Robins et al., 1994).

\section{MATERIALES Y MÉTODOS}

\section{Determinación de parámetros y fisicoquímicos de crecimiento microbiano en queso tipo Edam.}

Los parámetros se han determinado utilizando los modelos matemáticos Baranyi \& Roberts, Gompertz y Logístico. Durante el periodo de 24 días, se evaluaron en 8 partes de cada tratamiento (queso Edam elaborado en activación de cultivos iniciadores a temperaturas 30,38 y 45 $\left.{ }^{\circ} \mathrm{C}\right)$ como se puede observar en la Figura $\mathrm{N}^{\circ} 01$.

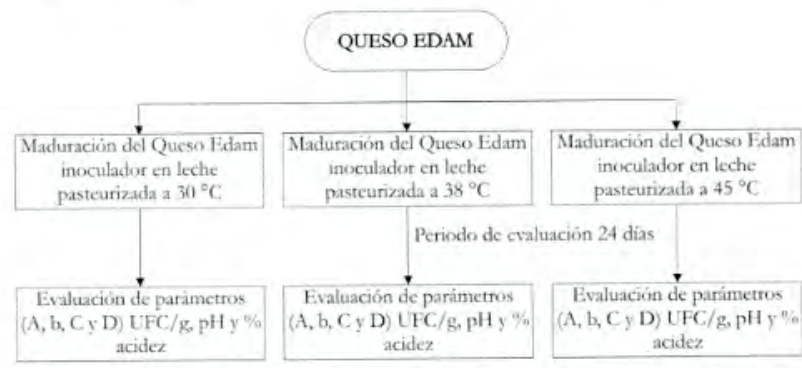

Figura $\mathrm{N}^{\circ} 01$. Diagrama de flujo para el estudio de $\mathrm{UFC} / \mathrm{g}$ y fisicoquímico

Los datos en crecimiento de (UFC/g) para cada temperatura fueron incorporados como variable dependiente en los modelos de crecimiento, mientras que el tiempo de crecimiento $(t)$ se incorporó como variable independiente. Se realizó un análisis de regresión con los modelos de Baranyi \& Robertz, Gompertz y Logístico por medio de algoritmo de Marquardt para encontrar en el ajuste los parámetros de regresión $\left(A, b, C_{\mathrm{y}} D\right)$.

$\log N=C+\ln \left(\left(-1+\exp \left(b^{*} D\right)+\exp \left(b^{*} t\right)\right) /\left(-1+\exp \left(b^{*} t\right)+\exp \left(\left(b^{*} D\right)+C \cdot A\right)\right)\right)$ $\log N=A+(C-A)^{*} \exp \left(-\exp \left(-b^{*}(t-D)\right)\right)$ $\log \mathrm{N}=A+\left((C-A) /\left(1+\exp \left(\left(4^{*} b^{*}(D-f) /(C-A)\right)+2\right)\right)\right)$

Donde:

$\log N$ : logaritmo común de la poblaciones bacterianas (UFC/mL)

$t \quad$ : periodo de incubación, es la variable independiente (horas)

A : logaritmo común de la población inicial en $\mathrm{UFC} / \mathrm{ml}$ (inóculo)

C : logaritmo común de la población final en la fase estacionaria

$b$ : representa la pendiente de la curva y describe la tasa de crecimiento 
D : tiempo en el cual la tasa de crecimiento es de mayor magnitud.

Utilizando los parámetros de ajuste de los modelos de Baranyi \& Robertz, Gompertz y Logístico, se calcularon la fase de adaptación ( $\lambda$ ), la tasa máxima de crecimiento exponencial ( $\mu$ max) y el tiempo de generación $(\mathrm{Tg})$, como descriptores microbiológicos a ser utilizados para efectos de predicción de la población en un tiempo dado y en cada temperatura incluida en el estudio $\left(30,38\right.$ y $\left.45^{\circ} \mathrm{C}\right)$, a tal efecto, se utilizaron las siguientes expresiones matemáticas:

Fase de latencia o adaptación $(\lambda) \quad: \quad \lambda=D-\frac{1}{b}$

Tiempodegeneración $(T g) \quad: \quad T_{g}=\log 2 \frac{e}{b C}$

Tasa especifica de crecimiento $\left(\mu_{\max }\right): \mu_{\max }=\frac{b C}{e}$

Para modelo matemático logístico : $\quad \lambda=D-\frac{2}{b}$

$$
T_{g}=\log 2 \frac{4}{b C}
$$

Validación de modelos matemáticos de crecimiento de cultivos iniciadores

\section{a) Modelamiento primario}

En esta etapa se usó los modelos primarios de Baranyi \& Roberts, Gompertz y Logístico para determinar la velocidad de crecimiento de cultivos iniciadores con el software STATGRAPHICS $®$ Centurion XVI para cada temperatura.

\section{b) Modelamiento secundario}

Obtenidas las máximas velocidades de crecimiento, se construyó un modelo secundario ajustando los datos de velocidad vs temperatura a una función matemática mediante regresión lineal. Posteriormente se obtuvo la ecuación que representa la relación. Esta expresión se convirtió en el modelo que se empleó para generar los datos de velocidad de crecimiento de cultivos iniciadores. Con el software Microsoft Excel 2010 y el software STATGRAPHICS $®$ Centurion XVI con la metodología de (Cabeza, 2013).

\section{c) Ajuste y validación del modelo}

La tercera etapa consiste en la validación estadística del modelo matemático con los datos obtenidos en el laboratorio y los datos generados por el modelo en la segunda etapa (Estimados). Para la validación se comparó estadísticamente el factor de sesgo (Bf), factor de exactitud (Af) y el Error cuadrado medio (RMSE) (García, et al., 2011) y (Judet-Correia et al., 2010).

Bf: el factor sesgo responde a la pregunta de si, en promedio los valores observados se encuentran por encima o por debajo de la línea de equivalencia y, en caso afirmativo, en qué medida.

Af: el factor exactitud mide la distancia mínima entre cada punto promedio y la línea de equivalencia como una medida de que tan cerca, en promedio se encuentran los valores predichos y los observados, por lo tanto, el Af es una medida de la desviación media y puede ser utilizado como una simple medida del nivel de confianza que se puede tener con el modelo de predicción.

RMSE: mide la desviación media entre los valores observados (Obs) y los valores estimados (Est) cuando menor sea el valor de este índice, mejor es el ajuste del modelo frente a los datos experimentales donde:

$$
\mu_{\max }=C_{o}-C_{1} T+C_{2} T^{2}
$$

$\mu_{\max } \quad$ : Máxima velocidad de crecimiento

$C_{\omega}, C_{\ell} y C_{2}$ : Coeficientes de la ecuación cuadrática

$T \quad:$ Temperatura

Factor de sesgo (B)

$$
B_{f}=10^{\left[\frac{\sum \log \left(\frac{\mu_{E s t}}{\mu_{\text {obs }}}\right)}{n}\right]}
$$

N : Número de observaciones usadas en el calculo

$\mu_{E=} \quad:$ Velocidad de crecimiento estimado

$\mu_{\text {obs }} \quad$ : Velocidad de crecimiento observado

\section{Factor de exactitud (A)}

$$
A_{f}=10\left[\frac{\sum \log \left(\frac{\mu_{E s t}}{\mu_{\text {obs }}}\right) \mid}{n}\right]
$$

Errorcuadrático medio (RMSE)

$$
R M S E=\sqrt{\frac{\sum\left(\mu_{E s t}-\mu_{o b s}\right)^{2}}{n-p}}
$$

n : Número de observaciones

$P \quad$ : Número de parámetros a ser estimados

$\mu_{E t t} \quad$ : Velocidad de crecimiento estimada

$\mu_{\text {obr }} \quad$ : Velocidad de crecimiento observada

\section{RESULTADOS Y DISCUSIÓN}

Determinación de parámetros del crecimiento de cultivos iniciadores durante la maduración en queso Edam con modelos matemáticos (Baranyi \& Roberts, Gompertz y Logístico).

El presente estudio se evaluó mediante tres modelos matemáticos (Baranyi \& Roberts), el desarrollo de cultivos iniciadores en agar M17 muestra los parámetros en las primeras 24 a 576 horas de adaptación y crecimiento los parámetros $A, b, C$ y $D$ ajustados con modelos matemáticos indican que a temperatura $30^{\circ} \mathrm{C}$ los valores son mayores y a temperatura 38 y $45^{\circ} \mathrm{C}$ menores (Tabla $\mathrm{N}^{\circ} 01$ ). Existe una elevada diferencia significativa entre las respuestas de crecimiento a las temperaturas y modelos matemáticos empleadas, siendo estos factores la causa del aceleramiento o retardo del crecimiento y ajuste con modelos. Existe una elevada correlación $R^{2}=96,955$ indicando la bondad de los modelos matemáticos que ajusta los datos experimentales para describir el comportamiento del crecimiento de cultivos iniciadores y que la desviación estándar indica con una tendencia bastante aceptable como se muestra en la Tabla $\mathrm{N}^{\circ} 01$.

En la Tabla $\mathrm{N}^{\circ} 01$ se presentan los parámetros $A, b$, 
Tabla $N^{\circ} 01:$ Parámetros obtenidos durante la maduración del queso Edam

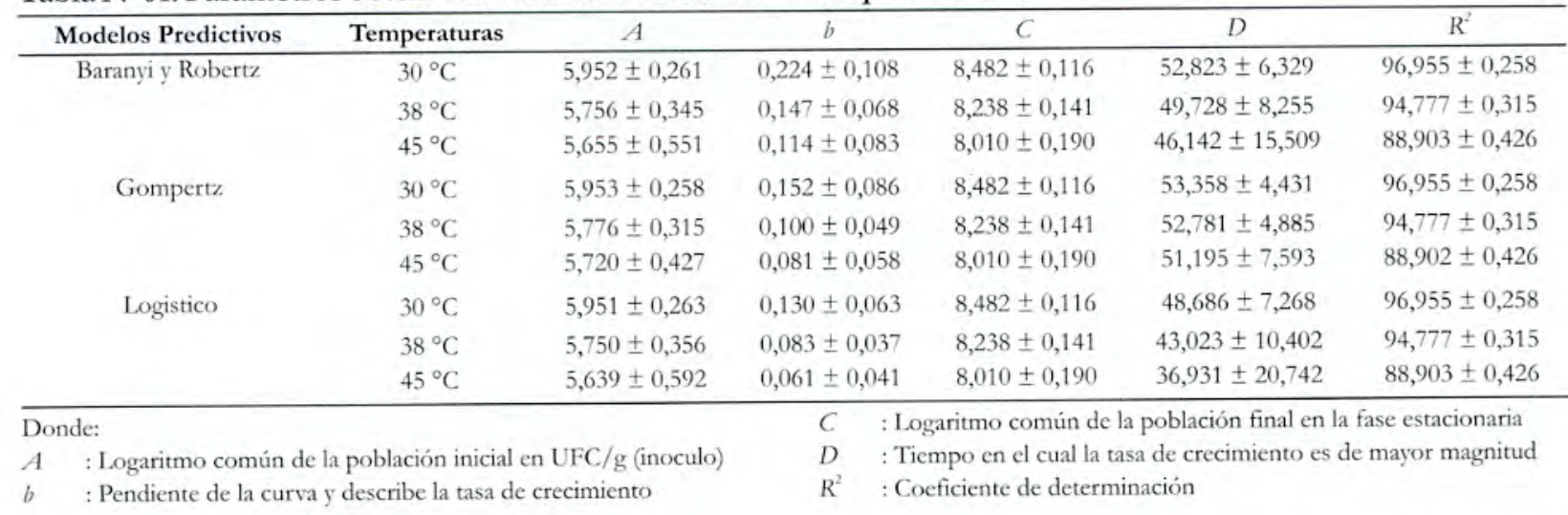

Cy $D$ ajustados al emplear las ecuaciones matemáticas para cada una de las temperaturas en estudio, se observa que a temperatura $30^{\circ} \mathrm{C}$ con los tres modelos, los parámetros $A$ y $C$ los valores coinciden, lo cual da una idea de similitud en el ajuste entre ellos, sin embargo los parámetros $b$ y $D$ muestra diferencia significativa entre los modelos con $b=0,224$; 0,152 y 0,$130 ; D=52,823 ; 53,358$ y 48,686 respectivamente. En este caso los modelos matemáticos que resultó con mejor ajuste fueron Baranyi \& Roberts y Gompertz, del mismo modo que encontró Zarate (2009) que determinó los parámetros del estudio del efecto de la temperatura sobre el crecimiento de Lactococcus lactis subs lactis, con parámetros $b=$ 0,51 y $C=5,82$ a temperatura $30^{\circ} \mathrm{C}$. Castro et al., (2008) encontró resultados similares estableciendo que los mejores predicciones del crecimiento de Lactococcus lactis subs lactis, en leche descremada en función de temperatura a 33 y $30^{\circ} \mathrm{C}$ la tasa de crecimiento general de 0,512 y 0,559 respectivamente. (Chacon, 1992) obtuvo resultados similares estableciendo que los mejores aislamientos del genero Lactococcus se efectúa a $\operatorname{los} 30{ }^{\circ} \mathrm{C}$ y la tasa de crecimiento general de $0,24 \mathrm{Log} \mathrm{UFC} / \mathrm{ml} / \mathrm{h}$. El estudio reporta que la temperatura óptima es de $30^{\circ} \mathrm{C}$, donde el crecimiento logaritmico de la población final en la fase estacionaria es de 8,482 Log UFC/g/h y el tiempo para alcanzar el máximo crecimiento fue de 336 horas. Las otras temperaturas ofrecieron menores valores, esto hace que indica, que no afectan de manera directa a los parámetros de crecimiento cinético.

La fase de latencia ( $\lambda$ ) y la velocidad máxima de crecimiento $\left(\mu_{\max }\right)$ se hacen mínimos en la medida que la temperatura se incrementa, mientras que el tiempo de generación $(T g)$ muestra un comportamiento inverso se muestra en la Tabla $\mathrm{N}^{\circ} 02$.

Respuestas parecidas tienen los parámetros velocidad máxima de crecimiento $\left(\mu_{\text {maco }}\right)$ y el tiempo de generación $(T g)$. Se destaca que la mejor expresión del crecimiento es a los $30^{\circ} \mathrm{C}$ en los cuales se registran tiempo de generación $(T g)$ de 0,635 horas, velocidad máxima de crecimiento $\left(\mu_{\text {max }}\right)$ de $0,699 \mathrm{Log} \mathrm{UFC} / \mathrm{g} / \mathrm{h}$ y la fase de latencia (入) que requiere para duplicarse es de 48,359 horas. El parámetro ( $\lambda$ ) 4,144 con el modelo Logístico, indica que a mayor temperatura la fase de latencia siempre es menor sin considerar que la activación de microorganismos tenga una determinada temperatura. Comparativamente encontramos que Yousef \& Carlstrom, (2006) reportan que la fase exponencial, el tiempo de generación $(T g)$ alcanza el valor más bajo y la velocidad máxima de crecimiento $\left(\mu_{\max }\right)$ el más elevado. Asimismo (Lade \& Chitanand, 2006) reporta valores de 1,89 horas, $1,16 \mathrm{Log} \mathrm{UFC} / \mathrm{ml} / \mathrm{h}$ y 0,22 horas respectivamente y (Cayré \& Garro, 2007) hallaron para los mismos parámetros los siguientes valores, 1,78 horas, 1,35 Log UFC $/ \mathrm{ml} /$ h y 0,19 horas estos datos permite afirmar que los ensayos realizados ofrecen respuestas fisiológicas bastante homogéneas de (Lactococcus lactis subs lactis, Lactococcus lactis subs cremoris y Streptococcus thermophilus) dentro su temperatura optima de crecimiento. A su vez, (Neumeyer et al., 1997) encontraron para el tiempo de generación una relación $\mathrm{GT}_{3, \mathrm{~T}} / \mathrm{GT}_{\mathrm{REC}}$ de 1,50 para Pseudomonas, trabajando con datos expresados en transmitancia (\%T). En otros estudios realizado, se han obtenido resultados similares;

Tabla $N^{\circ}$ 02: Parámetros cinéticos de crecimiento de cultivos iniciadores derivados de los modelos predictivos

\begin{tabular}{ccccc}
\hline Modelos Predictivos & Temperaturas & $\lambda$ & $T g$ & $\mu_{\text {mas }}$ \\
\hline Baranyi y Robertz & $30^{\circ} \mathrm{C}$ & 48,359 & 0,635 & 0,699 \\
& $38^{\circ} \mathrm{C}$ & 42,925 & 0,676 & 0,445 \\
Gompertz & $45^{\circ} \mathrm{C}$ & 37,37 & 0,896 & 0,336 \\
& $30^{\circ} \mathrm{C}$ & 46,779 & 0,635 & 0,474 \\
Logistico & $38^{\circ} \mathrm{C}$ & 42,781 & 0,993 & 0,303 \\
& $45^{\circ} \mathrm{C}$ & 38,849 & 1,261 & 0,239 \\
& $30^{\circ} \mathrm{C}$ & 33,301 & 1,092 & 0,406 \\
& $38^{\circ} \mathrm{C}$ & 18,927 & 1,197 & 0,252 \\
\hline
\end{tabular}

Donde:

$\lambda$ : Fase de latencia
Tg: Tiempo de generación

$\mu$ max : Velocidad máxima de crecimiento exponencial 
siendo la relación $\mu_{\mathrm{REC}} / \mu_{\mathrm{ABS}} 1,17$ y 1,05 y la relación $\lambda_{\mathrm{REC}} / \lambda_{\mathrm{ABS}}$ 1,40 y 1,24 para $S$. aureus en aerobiosis y anaerobiosis respectivamente (Castillejo, 2002).

En las Figuras $\mathrm{N}^{\circ} 02 \sim 04$, se presentan las curvas de ajuste obtenidas al representar gráficamente los valores presentados en la Tabla $\mathrm{N}^{\circ} 01$. En el mismo se aprecia como la línea de valores ajustados presenta buen ajuste entre los puntos obtenidos como parámetros de regresión $A$, calculado con los modelos primarios, para cada una de las temperaturas en estudio, en ellas se puede apreciar también la similitud en el ajuste de los diferentes modelos a los datos observados experimentalmente. Debido a que los modelos Baranyi \& Roberts y Gompertz resulta ser similar en el ajuste.

Castro et al., (2008), compararon los modelos Gompertz, Logístico, Stannard y Richars, encontrando que los modelos Logístico y Gompertz ajustaron bien a la data experimental, aunque concluyeron que el modelo de Gompertz fue el que presentó los mejores resultados para modelar el crecimiento del Lactococcus lactis subs. Lactis en leche estéril. Zwietering et al., (1990) compararon los modelos de Gompertz, Logístico, Stannard y Richards encontrando que todos los modelos ajustaron bien, aunque concluyeron que el modelo de Gompertz resulta suficiente para describir la curva de crecimiento bacteriano, debido, en parte, a su facilidad de ajuste. Quirino et al., (1999), utilizaron cuatro modelos sigmoidales Brody, Logístico, Gompertz y Richards, para caracterizar la circunferencia escrotal en toros Nellore en crecimiento, utilizando la metodología de López (2008), para comparar los diferentes modelos, encontrando mayor error, menor $R^{2}$ y mayor dificultad (número de iteraciones) para el modelo Richard y el mejor modelo en este estudio resultó el modelo Logístico. Cayré et al., (2007) compararon tres modelos primarios: Gompertz, Logístico y Baranyi, para describir la curva de crecimiento de bacterias lácticas y Brochothix sobre emulsiones cárnicas a fin de estimar los parámetros cinéticos de crecimiento: tiempo de latencia $(\lambda)$, máxima velocidad especifica de crecimiento $\left(\mu_{\max }\right)$, los modelos Gompertz y Baranyi produjeron los mejores ajuste en la mayoría de los casos presentados.

Validación de los modelos matemáticos (Baranyi \& Roberts, Gompertz y Logístico)

La validación matemática fue cuantificada por

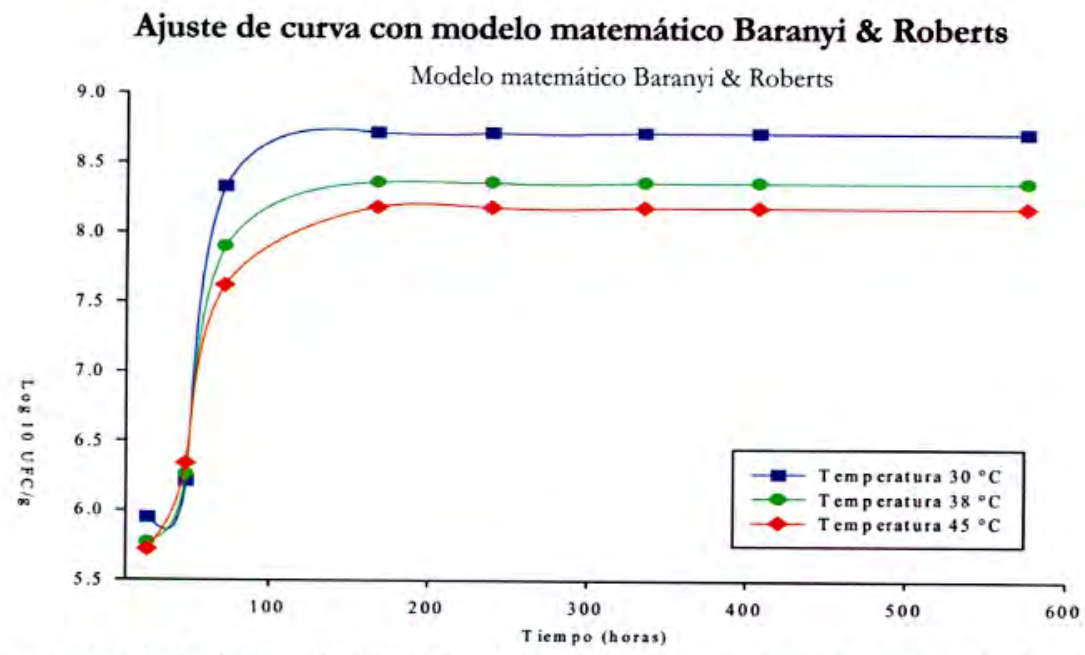

Figura $\mathbf{N}^{\circ}$ 02: Curvas de crecimiento cinético del Lactococcus lactis subs lactis, Lactococcus lactis subs cremoris y Streptococcus thermophilus a 30, 38 y $45^{\circ} \mathrm{C}$, con modelo Baranyi \& Roberts

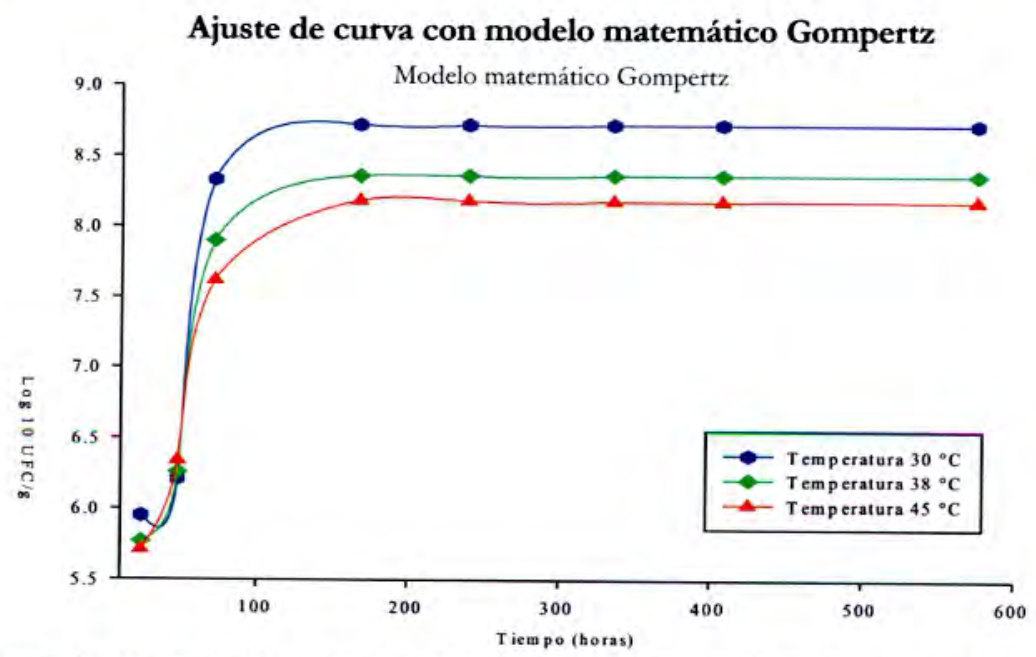

Figura $N^{\circ}$ 03: Curvas de crecimiento cinético del Lactococcus lactis subs lactis, Lactococcus lactis subs cremoris y Streptococcus 


\section{Ajuste de curva con modelo matemático Logístico}

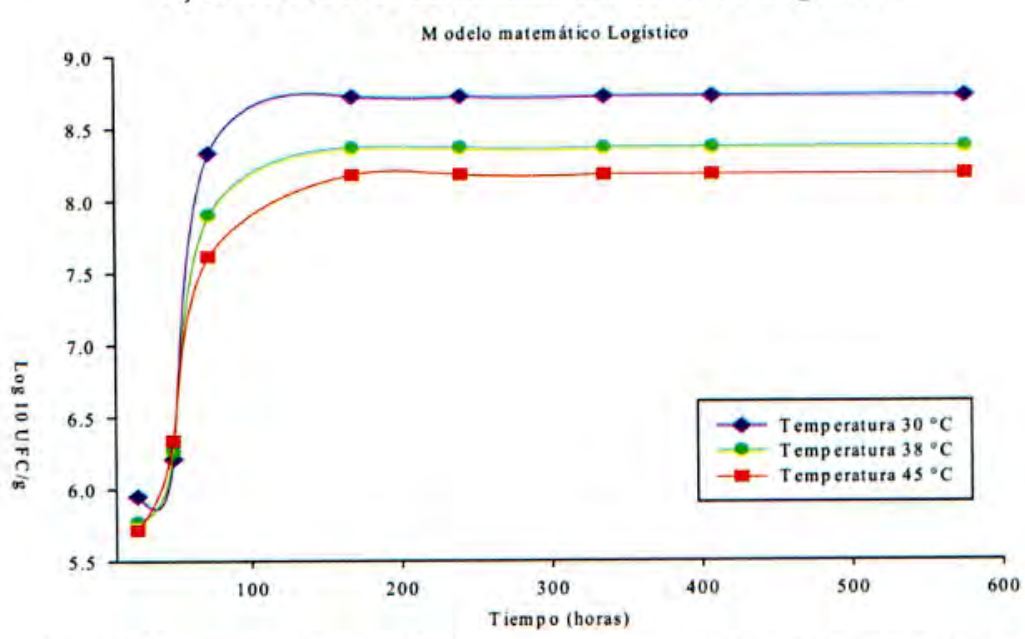

Figura $N^{\circ} 04$ : Curvas de crecimiento cinético del Lactococcus lactis subs lactis, Lactococcus lactis subs cremoris y Streptococcus thermophilus a 30,38 y $45^{\circ} \mathrm{C}$, con modelo Logístico.

medio del cálculo de los índices $A_{p}, B_{f}$ y RMSE, los cuales proporcionan un objetivo resumen de la fiabilidad de los modelos predictivos.

\section{Modelamiento primario}

En la Tabla $\mathrm{N}^{\circ} 03$, se muestra los datos experimentales de los tres modelos matemáticos sobre la velocidad máxima de crecimiento exponencial $\left(\mu_{\max }\right)$ y coeficiente de determinación $\left(R^{2}\right)$ a temperaturas 30,38 y 45 ${ }^{\circ} \mathrm{C}$. En este caso, el factor determinante en el crecimiento de cultivos iniciadores es la temperatura $30^{\circ} \mathrm{C}$, a mayor temperatura se produce una disminución de $\left(\mu_{\operatorname{mox}}\right)$ y la bondad de ajuste es de $R^{2}=0,970$, seguido para temperatura $38^{\circ} \mathrm{C} R^{2}=0,948$, finalmente a $45^{\circ} \mathrm{C} R^{2}=0,889$.

Tabla $N^{\circ} 03$. Velocidad máxima observada y error estándar del ajuste de los datos obtenidos para el crecimiento de cultivos iniciadores en función de la temperatura

\begin{tabular}{cccc}
$\begin{array}{c}\text { Modelos } \\
\text { Predictivos }\end{array}$ & Temperaturas & $\begin{array}{c}\boldsymbol{\mu}_{\max } \text { Obs(Ln } \\
\text { UFC/g*Horas) }\end{array}$ & $\mathrm{R}^{z}$ \\
\hline Baranyi y & $30^{\circ} \mathrm{C}$ & 0,699 & $0,970 \pm 2,58 \mathrm{E}-03$ \\
Robertz & $38^{\circ} \mathrm{C}$ & 0,445 & $0,948 \pm 3,15 \mathrm{E}-03$ \\
& $45^{\circ} \mathrm{C}$ & 0,336 & $0,889 \pm 4,26 \mathrm{E}-03$ \\
Gompertz & $30^{\circ} \mathrm{C}$ & 0,474 & $0,970 \pm 2,58 \mathrm{E}-03$ \\
& $38^{\circ} \mathrm{C}$ & 0,303 & $0,948 \pm 3,15 \mathrm{E}-03$ \\
& $45^{\circ} \mathrm{C}$ & 0,239 & $0,889 \pm 4,26 \mathrm{E}-03$ \\
Logistico & $30^{\circ} \mathrm{C}$ & 0,406 & $0,970 \pm 2,58 \mathrm{E}-03$ \\
& $38^{\circ} \mathrm{C}$ & 0,252 & $0,948 \pm 3,15 \mathrm{E}-03$ \\
& $45^{\circ} \mathrm{C}$ & 0,180 & $0,889 \pm 4,26 \mathrm{E}-03$ \\
\hline
\end{tabular}

\section{Modelamiento secundario}

La Tabla $\mathrm{N}^{\circ} 04$ presenta los resultados de velocidad máxima de crecimiento exponencial $\left(\mu_{\max }\right)$ de cultivos iniciadores por efecto de la temperatura, modelo secundario, generado mediante una función polinómica según la ecuación propuesta por García et al. (2011) y JudetCorreia et al. (2010).

\section{Validación de los modelos}

La validación matemática se ha realizado con datos
Tabla $N^{\circ} 04$ : Valores generados con programa Curve Expert mediante el ajuste de regresión cuadrática

\begin{tabular}{cccc}
\hline Coeficientes & Baranyi y Robertz & Gompertz & Logistico \\
\hline$C_{n}$ & 2,868 & 2,045 & 1,668 \\
$C_{1}$ & 0,104 & 0,077 & 0,060 \\
$C_{2}$ & 0,001 & 0,001 & 0,001 \\
\hline
\end{tabular}

experimentales obtenidos en condiciones de activación de cultivos a temperaturas de estudio, según la metodologia de estudio descrita por Cabeza, (2013) determinándose el factor el factor sesgo $(B)$, factor exactitud $(A)$ y raíz del error cuadrático medio (RMSE), como se observa en la Tabla $\mathrm{N}^{\circ} 05$.

En la Tabla $N^{\circ} 05$, se observa que los valores experimentales para $\left(\mu_{\max }\right)$ de cultivos iniciadores fueron, en general muy similares a los valores estimados por modelos predictivos. Los resultados obtenidos para el estadístico error cuadrado medio (RSME) mostraron desviaciones reducidas para los tres modelos, con valores de 0,$056 ; 0,000$ y 0,001 , respectivamente. Con relación a los valores de $B_{\rho}$ se observa que el modelo de Gompertz asociado estimó mejor velocidad máxima de crecimiento exponencial $\left(\mu_{\operatorname{mad}}\right)$, ya que presenta un valor de 1,000 , y para el resto de los modelos Baranyi \& Roberts y Logístico fueron próximos a $1 B_{f}=$ 1,1004 y 0,996 respectivamente. Estos índices, superiores o inferiores a la unidad, nos indica que las predicciones obtenidas con dichos modelos sobrestiman la $\left(\mu_{\max }\right)$, ya que los valores estimados son más elevados y bajos que los observados (ver Tabla $\mathrm{N}^{\circ} 06$ ). Esto indica que los valores estimados y observados para los modelos Baranyi \& Roberts y Gompertz se encuentran en la línea de equivalencia. No existe una gran diferencia entre los valores es decir, se encuentran muy próximas a la unidad y para el modelo Logístico los detalles presentan lo contrario.

En la literatura se ha encontrado numerosos estudios científicos que hacen referencia a los índices de validación matemática, $B_{f}$ y $A_{\rho}$ para velocidad máxima de crecimiento exponencial de diversos microorganismos. 
Tabla $\mathrm{N}^{\circ} 05$ : Tabla de frecuencias para la validación del modelo generado

\begin{tabular}{|c|c|c|c|c|c|}
\hline Modelos Predictivos & Temperaturas & $\mu_{H} \quad$ Obs (Ln UFC/g*Horas) & $\mu_{\text {L }}$ Est (Ln UFC $/ \mathrm{g}^{*}$ Horas) & Pre factor $B$ & $($ Est-Obs)^2 \\
\hline \multirow[t]{3}{*}{ Baranyi \& Roberts } & $30^{\circ} \mathrm{C}$ & 0,699 & 0,726 & 0,016 & $7,15 \mathrm{E}-04$ \\
\hline & $38^{\circ} \mathrm{C}$ & 0,445 & 0,489 & 0,040 & $1,88 \mathrm{E}-03$ \\
\hline & $45^{\circ} \mathrm{C}$ & 0,336 & 0,397 & 0,073 & $3,75 \mathrm{E}-03$ \\
\hline \multirow[t]{3}{*}{ Gompertz } & $30^{\circ} \mathrm{C}$ & 0,474 & 0,474 & $-0,0003$ & $1,64 \mathrm{E}-03$ \\
\hline & $38^{\circ} \mathrm{C}$ & 0,303 & 0,303 & $-0,0001$ & $4,19 \mathrm{E}-03$ \\
\hline & $45^{\circ} \mathrm{C}$ & 0,239 & 0,239 & 0,0006 & $8,19 \mathrm{E}-03$ \\
\hline \multirow[t]{3}{*}{ Logistico } & $30^{\circ} \mathrm{C}$ & 0,406 & 0,405 & $-0,001$ & $2,98 \mathrm{E}-07$ \\
\hline & $38^{\circ} \mathrm{C}$ & 0,252 & 0,251 & $-0,001$ & $7,04 \mathrm{E}-07$ \\
\hline & $45^{\circ} \mathrm{C}$ & 0,180 & 0,179 & $-0,003$ & $1,32 \mathrm{E}-06$ \\
\hline
\end{tabular}

Tabla $\mathrm{N}^{\circ} 06$ : Índice matemáticos y estadísticos obtenidos para los modelos de crecimiento de cultivos iniciadores

\begin{tabular}{lccc}
\hline \multicolumn{1}{c}{ Modelos Predictivos } & \multicolumn{3}{c}{ Índices Matemáticos } \\
& $B_{3}$ & $A_{f}$ & RMSE \\
\hline Baranyi \& Roberts & 1,104 & 1,104 & 0,056 \\
Gompertz & 1,000 & 1,000 & 0,000 \\
Logistico & 0,996 & 1,004 & 0,001 \\
\hline
\end{tabular}

Rodríguez (2003) realizó un estudio de los índices de validación en bacterias Aerobiosis y Anaerobiosis para la predicción de vida comercial de productos cárnicos, en el cual las estimaciones más ajustadas se obtuvieron $B_{f}=1,00 \mathrm{y}$ $A_{f}=1,09$ indicando que existe muy buen ajuste entre los valores experimentales y los valores estimado a partir de los modelos empleados. Barco (2001) realizó un estudio con $E$. coli 0157: $\mathrm{H7}$, en el cual las estimaciones más ajustadas se obtuvieron mediante el modelo de regresión $\left(B_{f}=0,88\right.$ y $A_{f}$ $=1,54)$. Para este mismo parámetro y el microorganismo patógeno S. aureus, Castillejo (2002) observó valores para los índices de validación de $B_{f}=1,13$ y $A_{f}=1,71$, mediante el modelo de Respuesta en Superficie en aerobiosis. Como se puede observar, los índices obtenidos en nuestro estudio se sitúan dentro del rango descrito por estos autores, e incluso son mejores, ya que se aproxima más a la unidad.

\section{CONCLUSIONES}

1. En el presente trabajo de investigación se concluye que la temperatura favorable para el crecimiento de cultivos iniciadores es $30^{\circ} \mathrm{C}$, ya que se activan y permite describir de mejor manera, el generación $(\mathrm{Tg})$ y velocidad máxima de crecimiento exponencial $\left(\mu_{\max }\right)$, que son indicadores para el manejo de los cultivos iniciadores.

2. El pH y acidez del queso Edam demuestran describir el crecimiento microbiano, a temperatura 30 y 38 ${ }^{\circ} \mathrm{C}$. Es posible que a temperaturas mayores a $38^{\circ} \mathrm{C}$, la flora acidoláctica se vea afectada por el descenso mínimo de $\mathrm{pH} \mathrm{e}$ incremento de acidez, reduciendo la población bacteriana.

3. Los modelos Baranyi \& Roberts y Gompertz mostraron predicciones exactas sobre la velocidad máxima de crecimiento exponencial $\left(\mu_{\mathrm{mix}}\right)$ y en cuanto a la validación de los modelos, el factor sesgo (Bf) y factor exactitud (Af) igualaron a la unidad, considerándose como seguros para describir los parámetros y ajuste de la curva de crecimiento. Sin embargo, para el modelo Logístico el factor sesgo (Bf) fue menor a uno, considerando un modelo regular para el ajuste de la curva de crecimiento.

\section{RECOMENDACIONES}

Se recomienda realizar la comparación de los modelos con mayor cantidad de datos y bajo diferentes condiciones de crecimiento, a fin de determinar cómo se comportan las unidades formadoras de colonias (UFC/g).

Se sugiere realizar estudios de la producción de lactocococina, pediocina y nisina que son inhibidores para los bacterias patógenos mediante la transformación de las bacterias de género lactococcus.

Se recomienda el estudio de vida útil de los alimentos procesados, con programas PMP, ComBase, DMFit y validación de los modelos matemáticos utilizando microbiología predictiva.

\section{REFERENCIAS BIBLIOGRÁFICAS}

Ayad, V. W., 2000. Application of wild starter cultures for flavour development in pilot plant cheese making, s.l.: Int Dairy J.

Baranyi, J. \& Roberts, T. A., 1994. A dynamic aproach to predicting bacterial growth in food. Food Microbiol, Volumen 23, pp. 277-294.

Barco, E. 2001. Tesis Doctoral. Elaboración de modelos predictivos de crecimiento microbiano para Escherichia coli O157:H7. Validación en productos cárnicos cocidos envasados al vacío. Universidad de Córdoba.

Cabeza, E. A. (2013). Desarrollo de un modelo matemático para evaluar el efecto de la temperatura sobre el crecimiento de S. Aureus en caldo BHI. Fundamentos de Microbiología Predictiva: Aplicaciones teóricas y prácticas, pp. 1-18.

Castillejo, A. (2002). Elaboración de modelos predictivos de crecimiento microbiano de Stapbylococcus aurens. Validación en productos cárnicos envasados al vacío.

Castro, G.; Valbuena, E.; Sánchez, E.; Briñez, W.; Vera, H.; Leal, M. (2008). Comparacion de modelos sigmoidales aplicados al crecimiento de Lactococcus lactis subsp. lactis. Revista Cientifica, FCV-LUZ, XVIII(5), pp. 582-588.

Cayré, E., Vignolo, M. \& Garro, A. (2007). Selección de un Modelo Primario para Describir la Curva de Crecimiento de Bacterias Lácticas y Brochotbrix thermosphacta sobre Emulsiones Cárnicas Cocidas. Informacion Tecnológica, 18(3), pp. 23-29.

Condori, C., 2010. Queseria Rural. $1^{\circ}$ ed. Juliaca: Lucero, Chacon, R. Z. (1992). Aislamiento e identificacion de bacterias ácido lácticas a partir de leche cruda de la zona alta Mérida. Tesis Biologo, Universidad de Los Andes. Facultad de Ciencias. Chacón, Z. \& López, G. (2000). Evaluacion de cepas de 
Lactococus como cultivos iniciadores en la elaboracion de quesos de pastaprensada. FCV-LUZ, X(5), pp. 423-428.

Garcia, D., Ramos, A. J., Sanchis, V. \& Marín, S. (2011). Modelling the effect of temperature and water activity in the gronth boundaries of Aspergillus ocbraceus and Aspergillus parasiticus. Food Microbiology, pp. 406-417.

Giffel, M. C. \& Zwietering, M. H., 1999. Validation of Predictive models describing the growth of Listeria monocitogenes. Int. J. Food Microbiol, Volumen 46, pp. 135-149.

Judet-Correia D, Bollaert S, Duquenne A, Charpentier C, Bensoussan M, Dantigny P. (2010). Validation of a predictive model for the gronth of Botrytis cinerea and Penicillium on grape berries. International journal Food Microbiology, pp. 106113.

Hill, A. R., 2007. Physical factors affecting flavour of Cheese. Woohead Publishing \& CRC Pres, pp. 252-283.

Holzapfel, W. F., 2002. Appropriate starter culture technologies for small-scale. Int. J. Food. Microbiol, Volumen 72, pp. 197-212.

Lade, H. S. \& Chitanand, M. P. (2006). Studies on some properties of bacteriocins produced by Lactobacillus species isolated from agrobased waste. The journal of Microbiology, II(1).

Lima, S., 2010. Ajuste a un modelo matemático, predicción y validación de un modelo experimental no-lineal. Instituto de Ingeniería de Alimentos para el Desarrollo.

López, S. 2006. Mejoras en el potencial reproductivo del rodeo. Selección por circunferencia escrotal. Diario Época. Suplemento Agro. [En línea]. Disponible en: http://www.diarioepoca.com/0000/00/00/index.php. [Consulta: 8 de octubre de 2008].

Madrid, A., 1994. Nuevo manual de tecnología quesera. Madrid España: Iraga.

McClure, P. J., Cole, M. B., Davies, K. W. \& Anderson, W. A., 1993. The use of automated turbidimetric data for the construction of kinetic models. J. Ind. Microbiol, Volumen 12,pp. 277-285.

Neumeyer, K., Ross, T., Thomson, G. \& McMeekin, T. A., 1997. Validation of a model describing the effects of temoerature and water activity on the growth of psychrotrophic psudomonasds. Food Microbiol, Volumen 38, pp. 55-63.

Oria, R., 1991. Elaboracion de productos lacteos. Zaragoza: Acribia.
Pavlidou, S., Bozoudi, D., Hatzikamari, M. \& Tzanetakis, N. (2011). Differentiation of Lactococcifrom 2 greek cheese with protecd designation of origen by phenotypic criteria and RAPD-PCR. Food Sci., pp. M175-M183.

Pin, C., Sutherland, J. P. \& Baranyi, J., 1999. Validating predictive models of food spoilage organisms. J. Appl. Microbiol, Volumen 87, pp. 491-499.

Quirino CR, Bergmann JA, Vale Filho VR, Andrade VJ, Pereira JC. (1999). Evaluation of four mathematical funtions to describe scrotal circumference maduration in Nellore bulls. Theriogenol, I(52), pp. 25-34.

Ramirez, M. A., 2005. Manual práctico de queseria. $1^{\circ} \mathrm{ed}$. Madrid: Ayala.

Robins, M., Brocklehurst, T. \& Wilson, P., 1994. Food structure and the growth of pathogenic bacteria. Food Technology International Europe, pp. 31-36.

Rodriguez, R. (2003). Desarrollo y validación de los modelos matemáticos para la predicción de vida comercial de productos cárnicos. Tesis Doctoral, p. 45.

Ross, T., 1996. Indices for performance evaluation of predictive models in food microbiology. J. Appl Bacteriol, Volumen 81,pp. 501-508.

Ross, T. \& McMeekin, T. A., 2003. Modelling microbial growth within food safety risk assessments. Risk Anal, Volumen 23,pp. 179-197.

Sandine, W., 1979. Lactis starter culture technology. In PfrizerChese.

Scott, R., Robinson, R. K. \& Wilbey, R. A., 2002. Fabricación de queso. $2^{\circ} \mathrm{ed}$. Zaragoza: Acribia.

Sutherland, J. P., Bayliss, A. J. \& Roberts, T. A., 1994. Predictive modelling of growth of Staphylococcus aureus: the effects of temperature, $\mathrm{pH}$ and sodium chloride. J. Food Microbiol, Volumen 21,pp. 217-236.

Van Boekel, M. A., 1996. Statistical aspcts of kinetic modellin for food science problems. J. Food Sci, Volumen 61, pp. 477-485.

Yousef, A. E. \& Carlstrom, C. (2006). Microbiologia de los Alimentos. Zaragoza: Acribia.

Zárate, E. (2009). Aplicación del modelo Cinético de Gompertz a la interacción del efecto de la temperatura. Ciencia y Tecnología, pp. 34-50.

Zwietering, M., Jongenburger, I., Rombouts, F. \& Vant Riet, K. (1990). Modeling of the bacterial growth curve. Appl Environ Microbiol, VI(56), pp. 1875-1881.

\section{Correspondencia:}

Edhy León Hancco: edhyleon@hotmail.com

Thomas Ancco Vizcarra: thoanviz@gmail.com
Fecha de Recepción: 20/06/2014

Fecha de Aceptación: 02/07/2014 